\title{
Complex Dielectric Function via the Kramers-Kronig Analysis in the Valence Electron Energy-Loss spectrum for $\mathrm{ZnTiO}_{3}$.
}

Juan Pantoja-Espinoza ${ }^{1}$, G. Herrera-Perez ${ }^{1}$, Carlos Ornelas-Gutiérrez ${ }^{2}$, Jesus Uribe-Chavira ${ }^{3}$, Miguel Meléndez-Zaragoza $^{3}$, Jesus Salinas-Gutiérrez ${ }^{3}$, Alejandro López-Ortiz ${ }^{3}$ and Virginia Collins-Martínez ${ }^{3}$

${ }^{1}$ Centro de Investigación en Materiales Avanzados, Chihuahua, Chihuahua, Mexico, ${ }^{2}$ Centro de Investigación en Materiales Avanzados, ${ }^{3}$ Centro de Investigación en Materiales Avanzados, United States

Electron energy-loss spectroscopy (EELS) is one of the most important techniques to elucidate the local electronic structure of materials. It has the advantage of covering the complete energy range, including valence inter-band transitions in the low energy-loss region, (VEELS); and core level (CEELS) excitations also known as energy-loss near-edge structure (ELNES) [1]. VEELS in a scanning transmission electron microscopy (STEM) mode also provides information about the bandgap energy Eg, and complex dielectric function. However, the VEELS-STEM analysis for perovskite zinc titanate $\left(\mathrm{ZnTiO}_{3}\right)$ with hexagonal phase has been rarely reported. This material is frequently used for optoelectronic and photocatalytic applications. The motivation of this work is to determine the bandgap energy Eg and to present the complex dielectric function for perovskite $\mathrm{ZnTiO}_{3}$ prepared by the Pechini method using the KramersKronig analysis in the VEELS.

$\mathrm{ZnTiO}_{3}$ was prepared by the Pechini method, the experimental details were reported elsewhere [2]. EELS spectra were obtained with an electron energy loss spectrometer (EELS GAT-777 STEMPack) attached to a JEM-2200FS $(200 \mathrm{kV})$, which offers an energy resolution of $1.0 \mathrm{eV}$. The electron probe size was below $1 \mathrm{~nm}$. The spectra were acquired using a dispersion of $0.05 \mathrm{eV} / \mathrm{channel}$ to record spectra up to 1000 $\mathrm{eV}$. The convergence semi-angle was $\alpha=9.0 \mathrm{mrad}$ for a $2.5 \mathrm{~mm}$ spectrometer entrance aperture and 40 $\mathrm{mm}$ camera length, and the corresponding collection semi-angle was $\beta=17.3 \mathrm{mrad}$. The deconvolution of the zero-loss peak from the VEELS region, the Fourier-log method to remove plural scattering, and Kramers-Kronig analysis were carried out using the Gatan Suite software [3].

Figure 1a shows the VEELS region where the zero-loss peak (ZLP) is identified and elemental analysis is performed for the $\mathrm{ZnTiO}_{3}$ that exhibits a hexagonal phase. Panel $b$ shows the ELNES for the Ti L2,3 edge identifying the split into $\mathrm{t} 2 \mathrm{~g}$ and eg orbitals due to the crystal field effects [3]. Panel c shows the brightfield STEM micrograph for $\mathrm{ZnTiO}_{3}$ morphology that exhibits particles with polygonal-shape. Subsequently, the ZLP was deconvoluted in the VEELS region considering in the fit the sum of a single Gaussian and a Lorentzian function squared [3]. The inelastic contribution spectrum was corrected for multiple scattering events using the Fourier-log method (see Figure. 2a) obtaining the single scattering distribution (SSD) [3]. It can be noticed that the bulk plasmon is located at $25.2 \mathrm{eV}$. The polynomial fit up to the $\mathrm{x}$-axis in the energy loss function (ELF) suggest an electronic bandgap (Eg) of $3.1 \mathrm{eV}$ (panel b). Panel c exhibits the complex dielectric function, $\varepsilon 1$ and $\varepsilon 2$. These curves were obtained through the Kramers-Kronig analysis [3].

It is demonstrated that the VEELS-STEM characterization combined with Kramers-Kronig analysis is a very useful technique to determine the electronic bandgap energy, Eg and to elucidate the complex dielectric function for perovskite $\mathrm{ZnTiO}_{3}$.

J. P-E thanks the CONACYT postdoctoral scholarship. G. H-P thanks to CONACyT-SEP Basic Research Project No. 253605. The authors gratefully acknowledge P. Pizá, E. Guerrero, K. Campos, R. Ochoa, and L. de la Torre (NANOTECH) for their facilities to complete the characterizations test. 

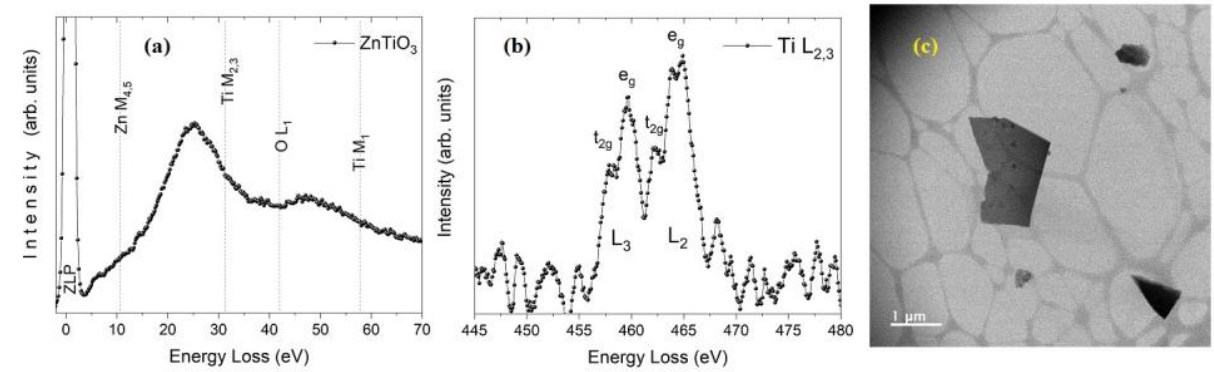

Figure 1. (a) Elemental identification in the VEELS region. (b) Ti L2,3-edge in the CEELS region. (c) Bright-field micrograph in STEM mode for perovskite $\mathrm{ZnTiO}_{3}$.
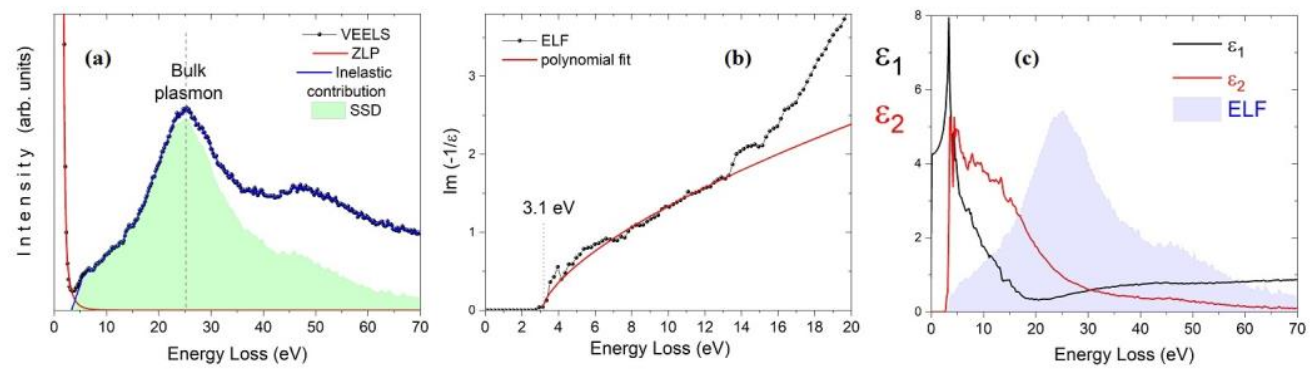

Figure 2. (a) Deconvolution of ZLP, inelastic contribution and SSD. (b) Polynomial fit in the ELF to determine the bandgap energy, Eg. (c) Complex dielectric function and ELF for $\mathrm{ZnTiO}_{3}$.

References

[1] G. Herrera-Pérez et al., J. Appl. Phys. 128 (2020), 064106.

[2] J. C. Pantoja-Espinoza et al., Catalysts 10 (2020), 1372.

[3] Digital Micrograph EELS Analysis User's Guide. Gatan, Inc. (2003) USA. 